\title{
Mannosylated lipoarabinomannan in serum as a biomarker candidate for subclinical bovine tuberculosis
}

\author{
Elise A Lamont ${ }^{1}$, João Ribeiro-Lima ${ }^{3}$, Wade Ray Waters ${ }^{4}$, Tyler Thacker ${ }^{4}$ and Srinand Sreevatsan ${ }^{1,2,5^{*}}$
}

\begin{abstract}
Background: Early and unambiguous detection of bovine tuberculosis (bTB), a significant disease of cattle worldwide, is necessary to control the spread of infection to other animals and humans. Current testing strategies are laborious, time consuming and heavily reliant on host responses that do not distinguish bTB from other mycobacteria. We report the presence of a pathogen signature, liparabinomannan (LAM), as a potential biomarker for bTB infection.

Findings: Fifty-five animals (uninfected [ $n=33]$, bTb [ $n=10]$ and exposed cases $[n=12]$ ) from a well characterized bovine serum repository were screened for the presence of LAM using a commercially available ELISA. Analysis showed that LAM had a sensitivity of $100 \%$ and a specificity of $91.7 \%$ for bTB detection (bTB positive versus bTB exposed animals).

Conclusion: LAM detection easily separated bTB infected animals from bTB exposed and negative controls. We propose that pathogen related markers, such as LAM, should be included with current testing strategies as a battery diagnostic for bTB.
\end{abstract}

Keywords: Bovine tuberculosis, Biomarker, Mycobacterium bovis, Cattle, Lipoarabinomannan, Diagnostics, Subclinical Infection, Serum

\section{Findings}

\section{Background}

Successful control of bovine tuberculosis (bTB) is achieved through the interplay of best management practices and diagnostics [1]. Early detection of infection informs quarantine procedures to limit disease spread and guides changes to the management plan for the future of the herd. However, current bTB diagnostic tools are labor intensive, lack combined sensitivity and specificity, and rely heavily on host responses, which cross-react with other mycobacteria and may result in the unnecessary culling of uninfected animals [2-6]. Advances in M. bovis functional genome usage during infection identified the release of mycobacterial specific proteins, peptides, DNA and lipids that are shed or secreted into host fluids $[7,8]$. We are now poised to capitalize on these biological processes

\footnotetext{
* Correspondence: sreev001@umn.edu

'Department of Veterinary Population Medicine, University of Minnesota,

St. Paul, Minnesota, USA

${ }^{2}$ Department of Veterinary Biomedical Sciences, University of Minnesota,

St. Paul, Minnesota, USA

Full list of author information is available at the end of the article
}

to develop diagnostics that center on the presence of a pathogen signature. In a previous study, we utilized three M. bovis specific peptides (Pks5, MB1895c, and MB2515c) identified in bTB infected sera as biomarkers for detection of subclinical infection [9]. We showed that all three $M$. bovis peptides were capable of detecting bTB infected animals and Pks5 had little to no cross-reactivity to other mycobacterial species [9]. It is important to note that the next-generation of $M$. bovis diagnostics is likely to follow a battery testing model, which includes multiple pathogen signatures, such as peptides and lipids. Therefore, we hypothesized that the major mycobacterial cell wall glycolipid, mannosylated lipoarabinomannan (ManLAM), also circulates in the serum and may be utilized as a biomarker for bTB infection. We validated the presence of ManLAM in randomly selected samples from a well characterized cattle serum repository including confirmed cases of M. bovis $(\mathrm{n}=10)$, bTB exposed (animals in contact with bTB positive cattle; $n=12)$ and negative controls $(n=33)$. Based on sensitivity and specificity analyses, we 
propose that ManLAM may aid current diagnostic tools for bTB infection.

\section{Methods}

\section{Sample source}

Field samples from bovine tuberculosis infected and exposed cases were generously provided by the National Veterinary Services Laboratory (NVSL; United States Department of Agriculture, Ames, IA) serum repositories. All field samples were collected from a single herd in California. Bovine tuberculosis disease status for each animal was validated using a combination of bacterial culture, antemortem caudal fold tests (CFT) and lesion histology at necropsy. Johne's disease history for this herd was indeterminate. Bovine tuberculosis exposed cases were defined as animals that had contact with bovine tuberculosis infected animals but remained bovine tuberculosis negative (i.e. negative results for culture, CFT and histology). Negative controls were collected from a bovine tuberculosis free dairy herd in Minnesota. Negative controls tested negative for $M$. bovis (culture, histology, and CFT).

\section{Lipoarabinomannan Enzyme-linked immunosorbent assay (ELISA)}

Randomly selected field sera samples (bTB positive $\mathrm{n}=$ 10 and bTB exposed $n=12)$, negative controls $(n=33)$, and negative controls spiked with either $M$. tuberculosis strain $\mathrm{H} 37 \mathrm{Rv}$ purified mannosylated lipoarabionmannan (ManLAM) (BEI Resources; NR-14848) or
M. smegmatis purified non-mannose-capped lipoarabinomannan (AraLAM) (BEI Resources; NR-14849) (Biodefense and Emerging Infections Research Resources Repository, NIAID, NIH) were diluted 1:5 in PBS and analyzed for the presence of LAM using the human LAM ELISA kit (Biotang, Waltham, MA) per manufacturer's instructions. A LAM standard curve was included using kit controls and two-fold dilution series of ManLAM spiked sera. The standard curve was plotted using GraphPad Prism software (GraphPad Software, La Jolla, CA). The optical density was read at $450 \mathrm{~nm}$ with a wavelength correction at $570 \mathrm{~nm}$. All samples were read in three wells. LAM ELISA was repeated twice.

\section{Statistical analysis}

Optical densities for each animal were averaged across the replicates. Receiver operating characteristic (ROC) curves were compared for 1) positive versus within-herd negative exposed (exposed) and 2) positive versus negative controls using the area under the ROC curves (AUC). Optimal cutoff values were determined by maximizing specificity and sensitivity by plotting the true negative rate against the true positive rate. ROC curves and specificitysensitivity plots were created in SPSS $^{\circ}$ (IBM Corp. Released 2013, IBM SPSS Statistics for Windows, Version 22.0, Amonk, NY).

\section{Results}

During the establishment and progression of $M$. bovis infection, mycobacterial specific proteins and lipids,

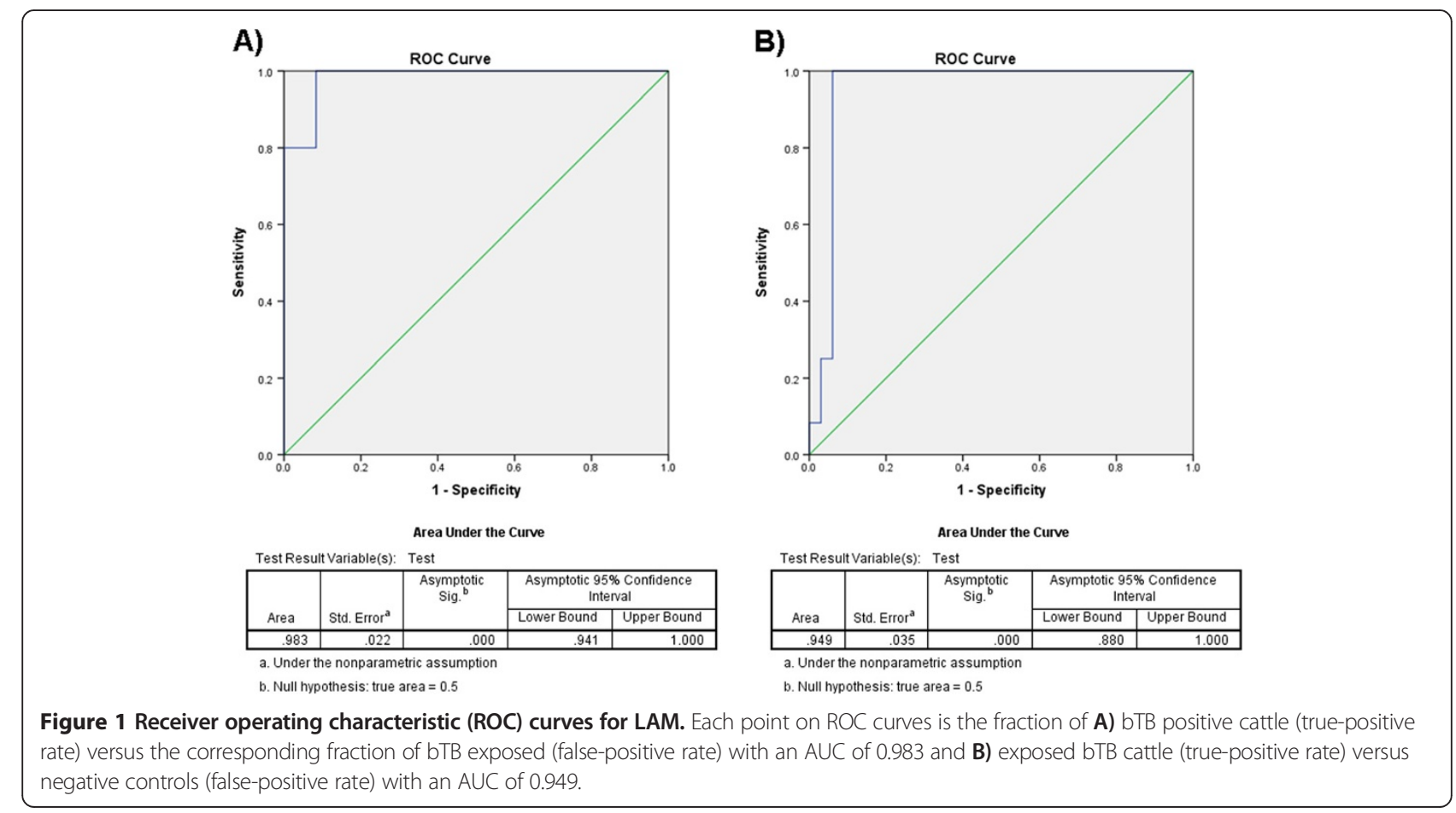


including those from the bacterial cell wall, are shed into host fluids. Several studies have shown the presence of lipoarabinomannan, a major cell wall glycolipid found on pathogenic mycobacteria, in sera and urine [10-13]. Therefore, we hypothesized that lipoarabionomannan may also be used as a biomarker to distinguish uninfected and $M$. bovis infected animals. Fifty-five animals (uninfected $[\mathrm{n}=33]$, bTb $[\mathrm{n}=10]$ and exposed cases $[\mathrm{n}=12])$ were screened for the presence of LAM using a commercially available ELISA. A LAM concentration curve was created and cross-reactivity to another glycolipid, non-capped lipoarabinomannan (AraLAM), found in environmental mycobacteria was assessed (Additional file 1). AraLAM was not discernable from the kit negative control (Additional file 1). Herds suspected of a bTB positive status will likely include contact animals (exposed) rather than pristine animals (i.e. uninfected and unexposed); therefore, ROC and AUC analyses compared
bTB positive and negative controls against bTB exposed animals. The AUC showed a near perfect separation for bTB positive versus bTB exposed animals (0.983) and bTB exposed versus negative control animals (0.949) (Figure 1A and B, respectively). Five cutoff ODs $(0.3368-0.16089 \mathrm{~nm})$ that retained high sensitivity and specificity were identified in BTB positive and exposed comparison by ROC analysis. ROC analysis of bTB exposed versus negative controls determined 3 cutoff ODs $(0.0055-0.068 \mathrm{~nm})$. The true positive and negative rates were plotted against the OD cutoffs to determine optimal cutoff values. The optimal cutoff value was calculated at $0.7901 \mathrm{~nm}$ with a sensitivity of $100 \%$ and a specificity of $91.7 \%$ for bTB positive versus bTB exposed animals (Figure 2A). The optimal cutoff value to distinguish bTB exposed animals from negative controls was calculated at 0.0055 with a sensitivity of $100 \%$ and specificity of $93.9 \%$ (Figure 2B). Together

A)

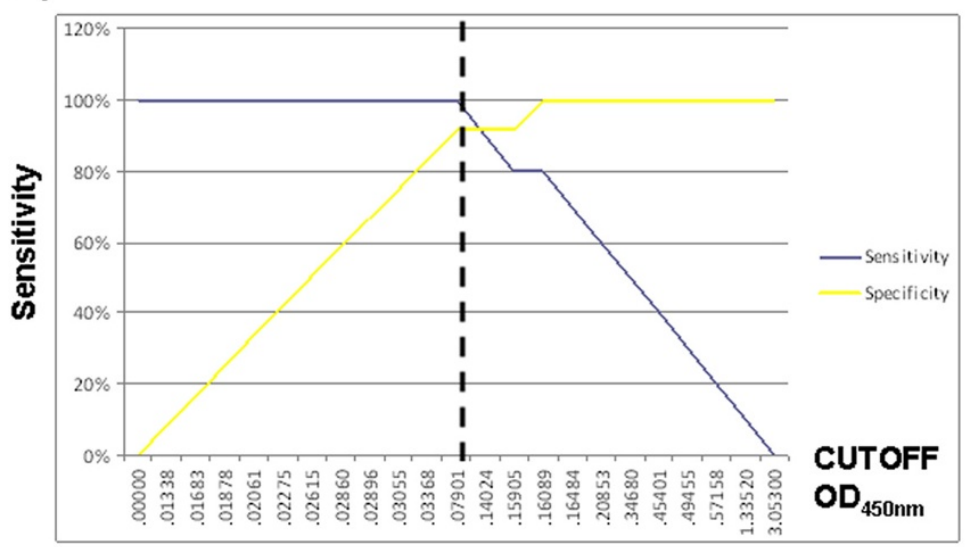

B)

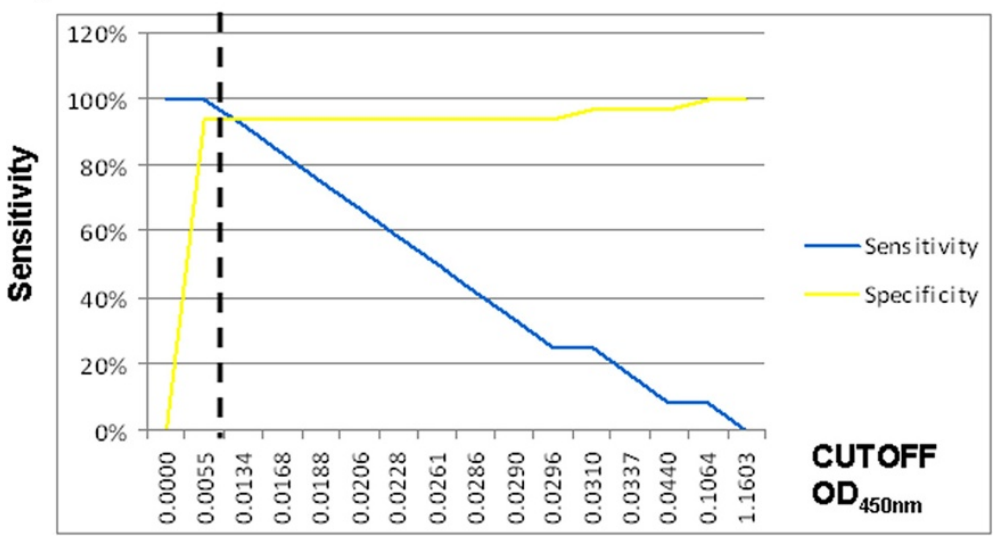

Figure 2 LAM distinguishes bovine tuberculosis positive and negative controls and exposed animals. The true negative rate and true positive rate using LAM as a biomarker were plotted against each other. The optimal cutoff value for A) bTB positive versus bTB exposed corresponded to O.D.450nm of 0.7901 (100\% sensitivity and $91.7 \%$ specificity) and B) bTB exposed versus negative controls corresponded to O.D.450nm of 0.0055 (100\% sensitivity and $93.9 \%$ specificity). Sensitivity = blue line and Specificity = yellow line. 
these data suggest that the LAM ELISA can be effectively applied for rapid detection of $M$. bovis infection and exposure.

\section{Discussion}

While detection systems utilizing host responses provided a first layer in identification of suspect diseased animals, they lack the needed specificity to eliminate cross-reactivity with other non-pathogenic mycobacteria or confusion with chronic illnesses of a non-infectious nature [3]. We have shown that mycobacterial peptides circulating in the serum accurately predict bTB infected from bTB negative and exposed animals [9]. We have extended our previous study to investigate another mycobacterial related component, LAM, likely present in serum of bTB infected animals, which may be used with additional pathogen signatures to provide a battery test for subclinical bTB. Traditionally, LAM is shed into the urine of infected hosts and is currently under investigation as a biomarker for human tuberculosis $[10,12,13]$. However, a recent study by Sakamuri et al. reported LAM coupled to high-density lipoprotein (HDL) in human tuberculosis positive sera [14]. It is important to note that LAM ELISA results may reflect exposure to environmental mycobacteria rather than show specificity for pathogenic mycobacteria. Therefore, we included sera spiked with AraLAM from M. smegmatis cultures and detected no significant changes to OD (Additional file 1). We show the presence of LAM correlates with bTb infection and is capable of separating infected from bTB exposed and negative animals. A single cutoff value applied to the true negative and positive rates showed LAM with a sensitivity of $100 \%$ and specificity of $91.7 \%$ for bTB positive versus bTB exposed animals (Figure 2). The identification of a glycolipid in infected serum also opens the possibility for identification of other pathogen related lipid biomarkers.

It is important to note that we analyzed a limited sample number for the presence of LAM. Although the results presented in this manuscript are promising for bTB diagnostics, a larger sample size will be required for validation. Furthermore, given the results reported by Sakamauri et al., pre-processing of sera involving HDL-nanodisc pulldown may be required to identify all bTB positive cases [14]. In addition to an increased sample size, sera from animals infected with other pathogenic mycobacteria, such as $M$. avium subsp. paratuberculosis, will need to be assessed to determine if cross-reactivity exists.

We propose that pathogen signatures, such as LAM, should be included in a two layer testing strategy for bTB. The first layer is a host related component in which the current strategies, newly identified host biomarkers, and LAM serve to identify suspected bTB positive animals such that a preliminary quarantine can be implemented [15]. The second layer of testing centers on a battery of circulating pathogen peptides to specifically distinguish bTB from other mycobacteria and chronic diseases [9].

\section{Conclusions}

LAM detection easily separated BTB infected animals from bTB exposed and negative controls. Future studies should include a larger sera sample size to validate LAM as a biomarker for bTB. We propose that pathogen related markers, such as LAM, should be included with current testing strategies as a battery diagnostic for bTB.

\section{Availability of supporting data}

The data sets supporting the results of this article are included within the manuscript and its additional files.

\section{Additional file}

Additional file 1: Lipoarabinomannan concentration curve. Only the positive control (Biotang kit-positive) and bovine sera spiked with LAM (0-300 ng/mL) showed a positive signal. Bovine sera spiked with non-capped lipoarabinomannan (AraLAM; M. smegmatis) did not react. All samples were conducted in triplicate.

\section{Abbreviations}

bTB: Bovine tuberculosis; CFT: Caudal fold test; LAM: Lipoarabinomannan; ManLAM: Mannosylated lipoarabionmannan; AraLAM: Non-mannose-capped lipoarabinomannan; ELISA: Enzyme-linked immunosorbent assay; ROC: Receiver operating characteristic; AUC: Area under the ROC curve; ODs: Optical densities; HDL: High density lipoprotein.

\section{Competing interests}

The authors declare that they have no competing interests.

\section{Authors' contributions}

EAL, JRL, WRW, and SS participated in the design of the work and interpretation of the results. WRW and TT provided infected field samples (bovine tuberculosis positive) and the National Veterinary Service Laboratories (NVSL, USDA-APHIS, Ames, IA) provided bovine tuberculosis negative exposed samples. EAL conducted ELISAs. JRL performed data analysis. EAL wrote the manuscript. All authors read and approved the final manuscript.

\section{Acknowledgements}

We thank the National Veterinary Services Laboratory, Ames, IA for its generous gift of bovine tuberculosis serum samples from their well-defined repositories. We thank the University of Minnesota Dairy Group for providing bovine tuberculosis negative control serum samples. Purified AraLAM and ManLAM were obtained through the $\mathrm{NIH}$ Biodefense and Emerging Infections Research Resources Repositories, NAID, NIH; Mycobacterium tuberculosis strain H37Rv, Purified LAM (NR-14848) and Mycobacterium smegmatis, Purified LAM (NR-14849). This study was supported by the Morris Animal Foundation Wildlife Fund (D14ZO-086) and the USDA (grant 61-4056A) awarded to S.S.

\section{Author details}

${ }^{1}$ Department of Veterinary Population Medicine, University of Minnesota, St. Paul, Minnesota, USA. ${ }^{2}$ Department of Veterinary Biomedical Sciences, University of Minnesota, St. Paul, Minnesota, USA. ${ }^{3}$ Faculdade de Medicina Veterinária, Universidade Lusósona de Humanidades e Tecnologias, Lisboa, Portugal. ${ }^{4}$ United States Department of Agriculture, National Animals Disease Center, Ames, lowa, USA. ${ }^{5}$ Veterinary Population Medicine and Veterinary Biomedical Sciences, College of Veterinary Medicine, University of Minnesota, 1971 Commonwealth Avenue, 55108 St. Paul, MN, USA.

Received: 25 April 2014 Accepted: 18 August 2014

Published: 21 August 2014 


\section{References}

1. Essey MA, Koller MA: Status of bovine tuberculosis in North America. Vet Microbiol 1994, 40:15-22.

2. Anonymous: Bovine Tuberculosis Eradication. In Edited by USDA. Washington, D.C: USDA, APHIS; 2005:35.

3. Kohler H, Gyra H, Zimmer K, Drager KG, Burkert B, Lemser B, Hausleithner D, Cubler K, Klawonn W, Hess RG: Immune reactions in cattle after immunization with a Mycobacterium paratuberculosis vaccine and implications for the diagnosis of $\mathrm{M}$. paratuberculosis and $\mathrm{M}$. bovis infections. J Vet Med B Infect Dis Vet Public Health 2001, 48:185-195.

4. Waters WR, Palmer MV, Thacker TC, Payeur JB, Harris NB, Minion FC, Greenwald R, Esfandiari J, Andersen P, McNair J, Pollock JM, Lyashenko KP: Immune responses to defined antigens of Mycobacterium bovis in cattle experimentally infected with Mycobacterium kansasii. Clin Vaccine Immunol 2006, 13:611-619.

5. Waters WR, Thacker TC, Nonnecke BJ, Palmer MV, Schiller I, Oesch B, Vordermeier HM, Silva E, Estes DM: Evaluation of gamma interferon (IFN-gamma)-induced protein 10 responses for detection of cattle infected with Mycobacterium bovis: comparisons to IFN-gamma responses. Clin Vaccine Immunol 2012, 19:346-351.

6. Whipple DL, Palmer MV, Slaughter RE, Jones SL: Comparison of purified protein derivatives and effect of skin testing on results of a commercial gamma interferon assay for diagnosis of tuberculosis in cattle. I Vet Diagn Invest 2001, 13:117-122.

7. Pollock NR, Macovei L, Kanunfre K, Dhiman R, Restrepo BI, Zarate I, Pino PA, Mora-Guzman F, Fujiwara RT, Michel G, Kashino SS, Campos-Neto A: Validation of Mycobacterium tuberculosis Rv1681 protein as a diagnostic marker of active pulmonary tuberculosis. J Clin Microbiol 2013, 51:1367-1373.

8. Green C, Huggett JF, Talbot E, Mwaba P, Reither K, Zumla Al: Rapid diagnosis of tuberculosis through the detection of mycobacterial DNA in urine by nucleic acid amplification methods. Lancet Infect Dis 2009, 9:505-511.

9. Lamont EA, Janagama HK, Ribeiro-Lima J, Vulchanova L, Seth M, Yang M, Kurmi K, Waters WR, Thacker T, Sreevatsan S: Circulating Mycobacterium bovis peptides and host response proteins as biomarkers for unambiguous detection of subclinical infection. J Clin Microbiol 2014, 52:536-543.

10. Gounder CR, Kufa T, Wada NI, Mngomezulu V, Charalambous S, Hanifa Y, Fielding K, Grant A, Dorman S, Chaisson RE, Churchyard GJ: Diagnostic accuracy of a urine lipoarabinomannan enzyme-linked immunosorbent assay for screening ambulatory HIV-infected persons for tuberculosis. J Acquir Immune Defic Syndr 2011, 58:219-223.

11. Patel VB, Singh R, Connolly C, Kasprowicz V, Zumla A, Ndungu T, Dheda K: Comparison of a clinical prediction rule and a LAM antigen-detection assay for the rapid diagnosis of TBM in a high HIV prevalence setting. PLoS One 2010, 5:e15664.

12. Shah M, Martinson NA, Chaisson RE, Martin DJ, Variava E, Dorman SE: Quantitative analysis of a urine-based assay for detection of lipoarabinomannan in patients with tuberculosis. J Clin Microbiol 2010, 48:2972-2974.

13. Shah M, Variava E, Holmes CB, Coppin A, Golub JE, McCallum J, Wong M, Luke B, Martin DJ, Chaisson RE, Martin EJ, Varaiva E, Dorman SE: Diagnostic accuracy of a urine lipoarabinomannan test for tuberculosis in hospitalized patients in a High HIV prevalence setting. J Acquir Immune Defic Syndr 2009, 52:145-151.

14. Sakamuri RM, Price DN, Lee M, Cho SN, Barry CE 3rd, Via LE, Swanson BI, Mukundan H: Association of lipoarabinomannan with high density lipoprotein in blood: implications for diagnostics. Tuberculosis (Edinb) 2013, 93:301-307.

15. Seth M, Lamont EA, Janagama HK, Widdel A, Vulchanova L, Stabel JR, Waters WR, Palmer MV, Sreevatsan S: Biomarker discovery in subclinical mycobacterial infections of cattle. PLoS One 2009, 4:e5478.

doi:10.1186/1756-0500-7-559

Cite this article as: Lamont et al: Mannosylated lipoarabinomannan in serum as a biomarker candidate for subclinical bovine tuberculosis. BMC Research Notes 2014 7:559.

\section{Submit your next manuscript to BioMed Central and take full advantage of:}

- Convenient online submission

- Thorough peer review

- No space constraints or color figure charges

- Immediate publication on acceptance

- Inclusion in PubMed, CAS, Scopus and Google Scholar

- Research which is freely available for redistribution

Submit your manuscript at www.biomedcentral.com/submit
C Biomed Central 